CDD: 617.6

\title{
PERFIL DA SAÚDE BUCAL E PRESENÇA DE CANDIDA NA CAVIDADE BUCAL DE PACIENTES ATENDIDOS NAS CLÍNICAS ODONTOLÓGICAS DA UEPG
}

\author{
ORAL HEALTH AND THE PRESENCE OF CANDIDA IN \\ THE ORAL CAVITY OF PATIENTS UNDER TREATMENT \\ IN DENTAL CLINICS OF UEPG
}

\author{
ELIZABETE BRASIL DOS SANTOS ${ }^{1}$ \\ HUMBERTO O. SCHWARTZ FILHO ${ }^{2}$ \\ EDSON ALBANO SCHWARTZ ${ }^{2}$ \\ ERIVAN S. RAMOS JÚNIOR ${ }^{2}$ \\ 1 Professora do Departamento de Odontologia da \\ UEPG \\ 2 Cirurgião-Dentista graduado na UEPG
}

\begin{abstract}
RESUMO
As doenças bucais mais freqüentes são causadas por microrganismos endógenos, que expressam seu potencial patogênico quando encontram condições favoráveis à sua proliferação. Para se verificar o perfil de saúde bucal e a presença de Candida na cavidade foram realizados exames clínicos intraorais, anamnese e coleta de saliva de 233 pacientes que procuraram atendimento nas clínicas odontológicas da UEPG. Dos 233 pacientes, 149 eram do sexo feminino e 84 do masculino, com média de idade de $33.21 \pm 12.30$ anos. As principais queixas desses pacientes foram "tratamento" e dor. O exame clínico demonstrou que $80 \%$ dos pacientes apresentavam higiene bucal regular ou deficiente. Candida foi isolada da
\end{abstract}

PUBLICATIO UEPG - Biological and Health Sciences, 8 (1): 57-73, 2002. 
cavidade bucal de 51,50\% dos pacientes e em maior quantidade naqueles usuários de próteses. Os resultados demonstram que os níveis de higiene bucal são baixos nesses pacientes; estes só procuram atendimento quando infecções como cárie e doença periodontal já estão instaladas e que o risco de desenvolvimento de infecções oportunistas, como a candidose é alto. Faz-se necessário que medidas de esclarecimento e incentivo sejam adotadas, para a manutenção da saúde bucal.

Palavras-chave: Candida; Candidose Bucal; saúde bucal

\section{Introdução}

A grande preocupação da classe odontológica na atualidade é com a prevenção de doenças infecciosas na cavidade bucal. Para o desenvolvimento de programas de prevenção faz-se necessário que se conheça as características da população com a qual se vai trabalhar. Dentre as infecções bucais, a candidose ocupa um lugar de destaque devido ao aumento nos casos dessa infecção, principalmente relacionados à condições imunossupressoras. O objetivo deste estudo foi traçar um perfil da saúde bucal da população que procura atendimento nas clínicas odontológicas da Universidade Estadual de Ponta Grossa, com ênfase na presença de Candida na cavidade bucal e de condições que possam favorecer o desenvolvimento de candidose bucal.

\section{Material e métodos}

Foram estudados 233 pacientes que passavam por triagem na Clínica de Estomatologia da UEPG. As alterações intra-orais foram verificadas mediante exame clínico e o status sistêmico através da anamnese. Para verificação da presença de Candida, a saliva, não estimulada, foi coletada em frascos esterilizados durante 1 minuto. A seguir as amostras foram homogeneizadas, diluídas em solução fisiológica até $10^{-3}$ e alíquotas de $0,1 \mathrm{~mL}$ foram semeadas em duplicata em placas contendo ágar Sabouraud Dextrose com cloranfenicol (Carlo Erba; $0.1 \mathrm{mg} / \mathrm{mL}$ de meio) e incubadas a $37^{\circ} \mathrm{C} / 48 \mathrm{~h}$. O número unidades formadoras de colônia por $\mathrm{mL}$ (ufc/mL) de Candida foi determinado mediante contagem do número de colônias carac- 
terísticas na melhor diluição. De cada placa positiva, foram isoladas até 3 colônias de Candida para posterior identificação das espécies. A identificação das espécies foi realizada segundo preconizado por Sandvén (1990) e Larone (1995), através da formação de tubo germinativo em soro estéril; clamidoconídio, em agar fubá; fermentação de carboidratos (zimograma) e assimilação de carboidratos (auxograma), para as demais espécies.

\section{Resultados}

\subsection{Perfil da população estudada observado através da anamnese e exame clínico}

Dos 233 pacientes, 149 eram mulheres e 84 homens, com média de idade de $33.21 \pm 12.30$ anos; sendo $90.12 \%$ com cor da pele branca, $1.71 \%$ parda e $8.15 \%$ negra. Quanto ao estado civil, 62.23\% eram casados, 30.04\% solteiros, $5.57 \%$ separados e $2.14 \%$ viúvos. Trabalhadores ativos constituíram 46,78\% dos pacientes (Tabelas 1 e 2). Quando questionados sobre o motivo que os levaram a procurar atendimento odontológico, os homens relataram, em ordem decrescente, tratamento, dor, revisão e confecção de prótese. Para as mulheres, dor foi o principal motivo relatado por $32.88 \%$ das pacientes, seguido de tratamento para $30.87 \%$ e revisão para $11.40 \%$ das pacientes (Tabela 3).

Tabela 1 - Distribuição dos pacientes quanto ao sexo e faixa etária (n=233)

\begin{tabular}{cccc}
\cline { 2 - 3 } & \multicolumn{2}{c}{ Sexo } & Total \\
\hline Faixa etária & Masculino & Feminino & $32(13.73 \%)$ \\
\hline < que 20 & $14(16.66 \%)$ & $18(12.08 \%)$ & $71(30.47 \%)$ \\
21 a 30 & $28(35.00 \%)$ & $43(28.85 \%)$ & $66(28.32 \%)$ \\
31a 40 & $23(27.38 \%)$ & $43(28.85 \%)$ & $47(20.17 \%)$ \\
41 a 50 & $14(16.66 \%)$ & $33(22.14 \%)$ & $09(3.86 \%)$ \\
51 a 60 & $01(1.19 \%)$ & $08(5.33 \%)$ & $05(2.57 \%)$ \\
61 a 70 & $03(3.57 \%)$ & $03(2.01 \%)$ & $02(0.85 \%)$ \\
> que 70 & $01(1.19 \%)$ & $01(0.67 \%)$ &
\end{tabular}


Tabela 2 - Distribuição dos pacientes quanto ao estado civil, cor da pele e ocupação $(n=233)$.

\begin{tabular}{cccc} 
& Masculino (n=84) & Feminino (n=149) & Total \\
\cline { 2 - 4 } Estado civil & $27(32.14 \%)$ & $43(28.85 \%)$ & $70(30.04 \%)$ \\
Solteiro & $54(64.28 \%)$ & $91(61.07 \%)$ & $145(62.23 \%)$ \\
Casado & $1(1.19 \%)$ & $4(2.68 \%)$ & $5(2.14 \%)$ \\
Viúvo & $2(2.38)$ & $11(7.38 \%)$ & $13(5.57 \%)$ \\
Separado & & & \\
Cor da Pele & $18(92.85 \%)$ & $132(88.59 \%)$ & $210(90.12$ \\
Brancos & $5(5.95 \%)$ & $14(9.19 \%)$ & $4(1.71 \%)$ \\
Pardos & & $49(32.88 \%)$ & $109(46.15 \%)$ \\
Negros & $60(71.42 \%)$ & - & $1(0.43 \%$ \\
Ocupação & $1(1.19 \%)$ & $88(59.06 \%)$ & $88(37.76 \%)$ \\
Trabalhador ativo & - & $12(8.05 \%)$ & $30(12.87 \%)$ \\
Aposentado & $18(21.42 \%)$ & & \\
Atividades Domésticas & & & \\
Estudantes ou desempregados & & & \\
\hline
\end{tabular}

Tabela 3 - Distribuição dos pacientes por sexo, de acordo com as queixas e motivos para a procura de atendimento na clínica odontológica $(\mathrm{n}=233)$

\begin{tabular}{lccc}
\hline $\begin{array}{c}\text { Queixas/ } \\
\text { Motivos }\end{array}$ & $\begin{array}{c}\text { Masculino } \\
(\mathbf{n = 8 4 )}\end{array}$ & $\begin{array}{c}\text { Feminino } \\
(\mathbf{n = 1 4 9 )}\end{array}$ & $\begin{array}{c}\text { Total } \\
(\mathbf{n}=\mathbf{2 3 3})\end{array}$ \\
\hline Tratamento & $30(35.71 \%)$ & $46(30.87 \%)$ & $76(32.61 \%)$ \\
Dor & $20(23.80 \%)$ & $49(32.88 \%)$ & $69(29.61 \%)$ \\
Revisão & $14(16.66 \%)$ & $17(11.40 \%)$ & $31(13.30 \%)$ \\
Prótese & $11(13.09 \%)$ & $16(10.73 \%)$ & $27(11.58 \%)$ \\
Dentes quebrados & $2(2.38 \%)$ & $10(6.71 \%)$ & $12(5.15 \%)$ \\
Estética & $6(7.14 \%)$ & $8(5.36 \%)$ & $14(6.00 \%)$ \\
Extração & $1(1.19 \%)$ & $3(2.01 \%)$ & $4(1.71 \%)$ \\
\hline
\end{tabular}

Através da anamnese observou-se que as alterações mais freqüentes relatadas pelos pacientes foram sangramento gengival e dificuldade de 
mastigação. Hábitos como tabagismo e etilismo foram de 3 a 4 vezes mais freqüentemente nos homens (Tabela 4).

Tabela 4 - Distribuição dos dados encontrados na anamnese dos pacientes, de acordo com o sexo ( $\mathrm{n}=233)$.

\begin{tabular}{lccc}
\hline \multicolumn{1}{c}{$\begin{array}{c}\text { Dados da } \\
\text { anamnese }\end{array}$} & $\begin{array}{c}\text { Masculino } \\
(\mathbf{n = 8 4 )}\end{array}$ & $\begin{array}{c}\text { Feminino } \\
(\mathbf{n = 1 4 9 )}\end{array}$ & $\begin{array}{c}\text { Total } \\
(\mathbf{n = 2 3 3 )}\end{array}$ \\
\hline Dificuldade de Mastigação & $28(33.33 \%)$ & $49(32.88 \%)$ & $77(33.04 \%)$ \\
Sangramento gengival & $34(40.47 \%)$ & $61(40.93 \%)$ & $95(40.77 \%)$ \\
Pouca abertura bucal & $14(16.66 \%)$ & $28(18.79 \%)$ & $42(18.02 \%)$ \\
Sede em excesso & $24(28.57 \%)$ & $37(24.83 \%)$ & $61(26.18 \%)$ \\
Tabagismo & $35(41.66 \%)$ & $22(14.76 \%)$ & $57(24.46 \%)$ \\
Etilismo & $22(26.19 \%)$ & $8(5.36 \%)$ & $30(12.87 \%)$ \\
Aftas freqüentes & $2(2.38 \%)$ & $23(15.43 \%)$ & $25(10.72 \%)$ \\
Diabetes & - & $2(1.34 \%)$ & $2(0.85 \%)$ \\
\hline
\end{tabular}

Como observado na tabela 5 , a maioria dos pacientes, tanto homens quanto mulheres, faziam higienização da cavidade bucal de 2 a 3x/dia. O exame clínico revelou que apenas $15,47 \%$ dos homens e 13,42\% apresentavam boa higiene bucal.

Tabela 5 - Relação entre o número de escovação/dia e classificação da higiene bucal através do exame clínico ( $\mathrm{n}=233)$.

\begin{tabular}{ccccccc} 
& \multicolumn{3}{c|}{ Masculino (n=84) } & \multicolumn{3}{c}{ Feminino (n=149) } \\
\cline { 2 - 7 } Escovação & Boa & Regular & Deficiente & Boa & Regular & Deficiente \\
\hline 1X/dia & - & - & $3(2.01 \%)$ & $1(1.19 \%)$ & - & $3(3.57 \%)$ \\
2X/dia & $4(2.68 \%)$ & $13(8.72 \%)$ & $13(8.72 \%)$ & - & $7(8.33 \%)$ & $14(9.39 \%)$ \\
3X/dia & $10(6.71 \%)$ & $52(34.89 \%)$ & $34(22.81 \%)$ & $10(11.90 \%)$ & $24(28.57 \%)$ & $13(8.72 \%)$ \\
4 ou + X/dia & $6(4.02 \%)$ & $10(6.71 \%)$ & $4(2.68 \%)$ & $2(2.38 \%)$ & $6(7.14 \%)$ & $4(2.68 \%)$ \\
\hline Total & $20(13.42 \%)$ & $75(50.33 \%)$ & $54(36.24 \%)$ & $13(15.47 \%)$ & $37(44.04 \%)$ & $34(40.47 \%)$ \\
\hline
\end{tabular}




\section{Presença de Candida na cavidade bucal}

A pesquisa de Candida foi realizada na saliva dos 233 pacientes. Candida foi isolada em 120 (51.50\%) pacientes, 46 (19.74\%) homens e 74 (31.75\%) mulheres. Dos 84 pacientes do sexo masculino, 54.76\% foram positivos para a levedura e dos 149 do sexo feminino, Candida foi isolada de 79.66\%. (Tabela 7).

Tabela 7 - Número de pacientes que apresentaram culturas da saliva positivas para Candida $(\mathrm{n}=233)$

\begin{tabular}{cccc}
\cline { 2 - 4 } Sexo & $\begin{array}{c}\text { Masculino } \\
(\mathbf{n = 8 4})\end{array}$ & $\begin{array}{c}\text { Feminino } \\
(\mathbf{n = 1 4 9 )}\end{array}$ & $\begin{array}{c}\text { Total } \\
(\mathbf{n = 2 3 3})\end{array}$ \\
\hline $\begin{array}{c}\text { Número de pacientes com } \\
\text { a presença de Candida na } \\
\text { cavidade bucal }\end{array}$ & $46(54.76 \%)$ & $74(49.66 \%)$ & $120(51.50 \%)$ \\
\hline
\end{tabular}

Considerou-se o paciente "negativo" quando não se observou crescimento da levedura nos meios de cultivo; "portadores" aqueles pacientes que apresentaram um número de ufc/mL de Candida inferior a 400 e "positivos", quando o número de ufc/mL foi superior a 400 . Como demonstrado na tabela 8, dos 120 pacientes, 61 (50.83\%) apresentaram até 400 ufc/ $\mathrm{mL}$ de Cândida. Número maior que $400 \mathrm{ufc} / \mathrm{mL}$ foi encontrado em 59 (49.16\%) pacientes.

Tabela 8 - Distribuição dos pacientes de acordo com o número de ufc/mL de Candida isolado da saliva.

\begin{tabular}{cccc}
\hline \multicolumn{2}{c}{$\begin{array}{c}\text { Masculino (n=46) } \\
\text { ufc/mL }\end{array}$} & \multicolumn{2}{c}{$\begin{array}{c}\text { Feminino (n=74) } \\
\text { Ufc/mL }\end{array}$} \\
\hline Até 400 & $>400$ & Até 400 & $>400$ \\
\hline $27(32.14)$ & $19(22.61)$ & $34(22.81)$ & $40(26.84)$ \\
\hline
\end{tabular}


Na tabela 9 estão distribuídos os pacientes com a presença de Candida e o número de ufc/mL, de acordo com a idade e sexo. Maior prevalência foi observada na idade entre 20 e 40 anos para ambos os sexos.

Tabela 9 - Distribuição dos pacientes de acordo com o sexo, idade e presença de Candida na cavidade bucal (negativos- $0 \mathrm{ufc} / \mathrm{mL}$; portadores- $<400 \mathrm{ufc} / \mathrm{mL}$ e positivos - > $400 \mathrm{ufc} / \mathrm{mL}$ ) $\mathrm{n}=233$

\begin{tabular}{|c|c|c|c|c|c|c|}
\hline \multirow[b]{2}{*}{ Faixa Etária } & \multicolumn{3}{|c|}{ Masculino } & \multicolumn{3}{|c|}{ Feminino } \\
\hline & Negativo & $\begin{array}{c}\text { ufc } / \mathrm{mL} \\
<400\end{array}$ & $\begin{array}{c}\mathrm{ufc} / \mathrm{mL} \\
>400\end{array}$ & Negativo & $\begin{array}{c}\mathrm{ufc} / \mathrm{mL} \\
<400\end{array}$ & $\begin{array}{l}\mathrm{ufc} / \mathrm{mL} \\
>400\end{array}$ \\
\hline$<$ que 20 & $5(5.95 \%)$ & $8(9.52 \%)$ & $1(1.19 \%)$ & $13(8.72 \%)$ & $5(3.35 \%)$ & - \\
\hline 21 a 30 & $14(16.66 \%)$ & $13(15.47 \%)$ & $1(1.19 \%)$ & $22(14.76 \%)$ & $16(10.73 \%)$ & $5(3.35 \%)$ \\
\hline 31 a 40 & $9(10.71 \%)$ & 13(15.47\%) & $1(1.19 \%)$ & $17(11.40 \%)$ & $17(11.40 \%)$ & $6(4.02 \%)$ \\
\hline 41 a 50 & $5(5.95 \%)$ & $6(7.14 \%)$ & $3(3.57 \%)$ & $18(12.08 \%)$ & $12(8.05 \%)$ & $3(2.01 \%)$ \\
\hline 51 a 60 & - & $1(1.19 \%)$ & - & $2(1.34 \%)$ & $3(2.01 \%)$ & $1(0.67 \%)$ \\
\hline 61 a 70 & - & $3(3.57 \%)$ & - & $1(0.67 \%)$ & $2(1.34 \%)$ & - \\
\hline$>$ que 70 & $1(1.19 \%)$ & - & - & - & $1(0.67 \%)$ & - \\
\hline Total & $34(40.47 \%)$ & $44(52.38 \%)$ & $6(7.14 \%)$ & $73(48.99 \%)$ & $56(37.58 \%)$ & $15(10.07 \%)$ \\
\hline
\end{tabular}

A presença de Candida foi relacionada ao uso de próteses, higiene bucal e uso de medicamentos. Na tabela 10 encontra-se o número de pacientes usuários de prótese e o número de UFC/mL de Candida encontrado na saliva. Dos pacientes de prótese portadores de Candida, 19 (22.61\%) eram homens e 48 (31.21\%) mulheres. Destes 67 pacientes, 4 homens e 12 mulheres apresentaram um número maior que $400 \mathrm{ufc} / \mathrm{mL}$ de Candida na cavidade bucal. Comparando-se o cuidado com a higiene bucal e a presença de Candida observamos que apenas em 8 (17.39) pacientes do sexo masculino e 8 (10.81\%) do sexo feminino portadores de Candida a higiene foi considerada boa (Tabela 11). 
Tabela 10 - Distribuição dos pacientes quanto ao uso de próteses e presença de Candida na cavidade bucal

\begin{tabular}{|c|c|c|c|c|}
\hline & Sexo & PT & PPR & $\mathrm{PF}$ \\
\hline & Neg & - & $04(21.05 \%)$ & 01(5.26\%) \\
\hline Masculino & $0<400 \mathrm{ufc} / \mathrm{mL}$ & 03 (15.78\%) & 05(26.31\%) & 02(10.52\%) \\
\hline \multirow[t]{2}{*}{$(n=19)$} & $>400 \mathrm{ufc} / \mathrm{mL}$ & 015.26\%) & - & 03(15.78\%) \\
\hline & Neg & - & 03(6.25\%) & $06(12.50 \%)$ \\
\hline Feminino & $<400 \mathrm{ufc} / \mathrm{mL}$ & 17(35.41\%) & 06(12.50\%) & 04(8.33\%) \\
\hline$(n=48)$ & $>400 \mathrm{ufc} / \mathrm{mL}$ & 7(14.58\%) & 02(4.16\%) & $03(6.25 \%)$ \\
\hline
\end{tabular}

PT - Prótese total

PPR - Prótese parcial removível

PF - Prótese fixa

Tabela 11 - Distribuição dos pacientes de acordo com o número de UFC/mL de Candida e presença de fatores predisponentes à candidose bucal $(\mathrm{n}=120)$

\begin{tabular}{lcccccc}
\cline { 2 - 7 } & \multicolumn{3}{c}{$\begin{array}{c}\text { Masculino (n=46) } \\
\text { ufc/mL }\end{array}$} & & \multicolumn{3}{c}{$\begin{array}{c}\text { Feminino (n=74) } \\
\text { ufc/mL }\end{array}$} \\
\hline Higiene Bucal & $>400$ & $<400$ & Total & $>400$ & $<400$ & Total \\
\cline { 2 - 7 } Boa & $7(15.21 \%)$ & $1(2.17 \%)$ & $8(17.39 \%)$ & $4(5.40 \%)$ & $4(5.40 \%)$ & $8(10.81 \%)$ \\
Regular & $10(21.73 \%)$ & $11(23.91 \%)$ & $21(45.65 \%)$ & $19(25.67 \%)$ & $18(24.32 \%)$ & $37(50.00 \%)$ \\
Deficiente & $10(21.73 \%)$ & $7(15.21 \%)$ & $17(36.95 \%)$ & $11(14.86 \%)$ & $18(24.32 \%)$ & $29(39.18 \%)$ \\
Total & $27(58.69 \%)$ & $19(41.30 \%)$ & $46(100 \%)$ & $34(45.94 \%)$ & $40(54.05 \%)$ & $74(100 \%)$ \\
\hline
\end{tabular}

Houve predominância de pacientes portadores de Candida quando relacionamos a sua presença com boca seca, hábitos nocivos como álcool e tabaco (Tabela 12). 
Tabela 12 - Distribuição dos pacientes de acordo com a queixa de boca seca, hábitos nocivos como álcool e tabagismo e presença de Candida na cavidade bucal $(\mathrm{n}=233)$

\begin{tabular}{lr|cc}
\cline { 3 - 4 } & \multicolumn{2}{c}{ Masculino } & Feminino \\
\hline \multirow{2}{*}{ Boca seca } & $<400 \mathrm{ufc} / \mathrm{mL}$ & $12(21.00 \%)$ & $11(19.29 \%)$ \\
$(\mathrm{n}=57)$ & $>400 \mathrm{ufc} / \mathrm{mL}$ & $01(1.75 \%)$ & $05(8.77 \%)$ \\
& Negativo & $08(26.66 \%)$ & $04(13.33 \%)$ \\
Álcool & $<400 \mathrm{ufc} / \mathrm{mL}$ & $11(36.66 \%)$ & $05(16.66 \%)$ \\
$(\mathrm{n}=30)$ & $>400 \mathrm{ufc} / \mathrm{mL}$ & $02(6.66 \%)$ & - \\
& Negativo & $11(19.64 \%)$ & $10(17.85 \%)$ \\
Tabagismo & $<400 u f c / m L$ & $19(33.92 \%)$ & $06(10.71 \%)$ \\
$(\mathrm{n}=56)$ & $>400 \mathrm{ufc} / \mathrm{mL}$ & $05(8.92 \%)$ & $05(8.92 \%)$ \\
\hline
\end{tabular}

Foram isoladas 312 colônias de Candida para identificação das espécies. Das 123 colônias isoladas dos pacientes do sexo masculino, e 189 isoladas do sexo masculino, 77 (62.60\%) e 109 (57.67\%) respectivamente foram de C. albicans. As demais foram identificadas em ordem decrescente como C. tropicalis, C. parapsilosis e C. krusei.

\section{Discussão}

O número de infecções oportunistas aumentou consideravelmente nos dias atuais e está relacionado com o grande número de condições imunossupressoras, caracterizadas pelo aumento no número de casos de pacientes com AIDS e pacientes submetidos à terapias imunossupressoras, como transplantados (HEIMDAHL \& NORD, 1990). Dentre essas infecções oportunistas, a candidose bucal merece destaque na odontologia, uma vez que cabe ao cirurgião dentista prevenir, tratar e identificar nos seus pacientes a presença de 
condições predisponentes à implantação, multiplicação e desenvolvimento de infecção pela Candida. Estudos epidemiológicos que buscam identificar indivíduos portadores de Candida numa população são importantes como instrumentos para esses profissionais da saúde, na prevenção da candidose bucal.

Neste estudo, buscou-se identificar indivíduos portadores de Candida na população que procurou atendimento odontológico nas clínicas da Universidade Estadual de Ponta Grossa. Dos 233 pacientes estudados, 51.50\% apresentaram Candida na cavidade bucal, destes, 54.70\% eram do sexo masculino e $49.66 \%$ do sexo feminino. Na literatura observa-se uma variação de 10 a 70\% dos portadores de Candida em indivíduos sadios. Essa variação pode ser explicada pelos diferentes métodos de coleta, origem das amostras, meios de cultura, grupos de indivíduos estudados e métodos de análise (GERGELI \& URI, 1961; WILLIAMSON, 1972; BURDFORDMASON et al., 1988; STENDERUP, 1990; FOTOS et al., 1991; MARSH et al., 1992; WEBB et al., 1998). A presenças de Candida foi maior em indivíduos entre 21 e 40 anos de idade.

Os pacientes foram considerados positivos para Candida na cavidade bucal quando apresentaram valores superiores a $400 \mathrm{ufc} / \mathrm{mL}$ de Candida na saliva e portadores quando esses valores foram inferiores a 400 . Valores acima de $400 \mathrm{ufc} / \mathrm{mL}$ predominaram nos pacientes do sexo feminino (26.84\%) comparados com $22.61 \%$ no sexo masculino, embora os valores não tenham diferença estatística.

A xerostomia é considerada um dos mais importantes fatores predisponentes à candidose bucal, por alterar o equilíbrio da microbiota bucal, por privar os pacientes da ação mecânica da saliva na remoção de microrganismos da boca e pela ausência de constituintes salivares antimicrobianos, como anticorpos e enzimas responsáveis pelo controle da microbiota bucal (NAVAZESH et al., 1992; NARHI et al., 1993). Neste estudo a avaliação da xerostomia foi subjetiva; a sensação de boca seca foi determinada durante anamnese. Dos 233 pacientes, 24.47\% relataram a sensação de boca seca e necessidade de beber água com freqüência. Destes, $5.57 \%$ dos homens e $6.68 \%$ das mulheres apresentaram Candida na cavidade bucal, sendo que valores acima de $400 \mathrm{ufc} / \mathrm{mL}$ estavam presentes em apenas $0.45 \%$ dos homens e $2.14 \%$ das mulheres. Do total de pacientes, $9.44 \%$ dos homens e $15.02 \%$ das mulheres relataram sensação de boca seca. Maior número de pacientes do sexo feminino com xerostomia também foi relatado por Pavinen \& Larmar, 1982; Nahi et al., 1992; Billings et al., 
1996; Vissink et al., 1996; Nederfors et al., 1997.

A principal causa de indução de diminuição do fluxo salivar á a utilização de medicamentos com potencial xerostômico, entretanto neste estudo não houve relação entre xerostomia e medicamentos. O número de mulheres utilizando medicamentos foi maior que o número de homens e as mulheres utilizaram mais medicamentos que os homens, o que está em concordância com outros autores (MILLER et al., 1992).

Talvez o fator mais freqüentemente relacionado com o aumento dos níveis de Candida intra-bucal e na prevalência de candidose seja o uso de próteses, especialmente totais, mal adaptadas e mal higienizadas (ARENDORF \& WALKER, 1980; BERDICEVSKY et al., 1980; MARSH et al., 1992; NARHI et al., 1993; NANETTI et al., 1993; OHMAN et al., 1995; WEBB et al., 1998). Pode-se destacar co-fatores, como uso noturno e contínuo das próteses, microporosidades e superfície áspera na resina, material utilizado na confecção das próteses, irritação e reação alérgica ao acrílico, redução do fluxo salivar e alterações no $\mathrm{pH}$ sob a área coberta, traumatismos e outras alterações epiteliais pelo uso de próteses com favorecimento da aderência de microrganismos. Esses fatores causam alterações na face interna das próteses ou na superfície epitelial que favorecem a colonização e proliferação de Candida ou a ação de seus produtos e toxinas, determinando o aparecimento de mucosite (BUDTZ-JORGENSEN et al., 1972; BUDTZ-JORGENSEN et al., 1990; IACOPINO E WATHEN, 1992; NARHI et al., 1993; BLAIR et al., 1995). A presença de próteses foi verificada em $28.75 \%$ dos pacientes, $12.01 \%$ eram portadores de próteses totais, $8.58 \%$ de próteses parciais removíveis e $8.1 \%$ próteses fixas. Dos 28 pacientes usuários de próteses fixas, todos eram portadores de Candida na boca, sendo que em $28.54 \%$, o número de ufc/mL de Candida isolado foi maior que 400 . O uso de próteses totais, juntamente com higienização precária da cavidade bucal pode favorecer a permanência de Candida na boca e o desenvolvimento de candidose (IACOPINO \& WATHEN, 1992; KULAK et al., 1997). A higiene deficiente das próteses propicia o aumento dos níveis de Candida na placa associada à prótese, sua colonização e o conseqüente desenvolvimento de mucosites (BUDTZ-JORGENSEN et al., 1975; NARHI et al., 1995; BLAIR et al., 1995).

Observou-se ainda que o número de escovações dos dentes por dia não correspondia a uma melhor ou pior higienização da cavidade bucal. Dos 84 pacientes do sexo masculino, 55.95\% relataram escovar os dentes 
de 3 a 4 vezes por dia e dos 149 pacientes do sexo feminino, esses valores foram de 67.11\%; enquanto que nos exames clínicos da cavidade bucal, a higiene foi considerada regular ou deficiente para esses pacientes. Esses dados confirmam relatos de que opiniões subjetivas e avaliação objetiva da higienização bucal apresentam resultados conflitantes (HOVEN-CHUNG, 1989; PIETROKOVSKY et al., 1995). Esses resultados demonstram que a população necessita de esclarecimentos quanto às técnicas de escovação, o que pode ser conseguido através de campanhas realizadas pelas universidades de odontologia.

O percentual de pacientes portadores e positivos para Candida entre os fumantes foi de $62.5 \%$, mostrando relação entre o hábito de tabagismo e a presença de Candida. O aumento da queratinização do epitélio em pacientes fumantes parece facilitar a aderência facilitar a aderência de Candida, promovendo consequentemente infecções por Candida na mucosa bucal e talvez até uma variedade de espécies (ARENDORF et al., 1982; ARENDORF \& WALKER, 1984; CROCKETT et al., 1992; RINDUM et al., 1994; SAKKI et al., 1997; HOLMSTRUP, 1998). Alguns autores não encontraram relação entre o hábito de tabagismo e o aumento do número de portadores de Candida (BASTIAN \& READE, 1982; OLIVER \& SHILIOTOE, 1984; STENDERUP, 1990).

Embora existam meios cromógenos e kits para identificação de espécies de Candida, as provas de formação de tubo germinativo, clamidioconídio, fermentação e assimilação de carboidratos ainda são os testes mais utilizados para sua identificação (SANDVÉN, 1990, WEBB et al., 1998). Em nosso estudo Candida estava presente em $51.50 \%$ dos pacientes. C. albicans foi a espécie mais prevalente, correspondendo a 59.61\% das amostras isoladas. Dos homens foram isoladas 123 colônias de Candida e 77 destas correspondera à C. albicans; das mulheres foram isoladas 189 colônias, sendo 109 de C. albicans. Esses achados correspondem aos dados relatados por vários autores sobre a prevalência da $C$. albicans sobre as demais espécies na colonização da cavidade bucal e no desenvolvimento de candidose (RAMIREZ-AMADOR et al., 1997; KINDELAN et al., 1998).

Os resultados encontrados neste estudo podem indicar a grande prevalência de portadores de Candida na população e sua divulgação pode servir como auxílio para a prevenção e controle da candidose bucal. Observamos ainda a importância dos cuidados com higiene bucal na prevenção da candidose e da manutenção da saúde bucal como um todo e que hábitos 
nocivos como tabagismo e alcoolismo podem favorecer a implantação da levedura na cavidade bucal e o desenvolvimento da infecção.

\title{
5. Conclusões
}

1- A população que procura atendimento nas clínicas odontológicas da UEPG necessita de maiores esclarecimentos sobre manutenção da saúde bucal. Campanhas de esclarecimento devem ser realizadas. As Universidades têm um papel importante para que o quadro observado neste estudo seja revertido.

2- Foi alta a prevalência de Candida na população estudada, principalmente relacionada ao uso de próteses. C. albicans foi a espécie mais prevalente.

3- Os pacientes estudados só procuraram atendimento odontológico quando da presença de lesões cariosas ou presença de dor. Não existe na população estudada a consciência da adoção de medidas preventivas contra infecções bucais.

\begin{abstract}
The most common oral diseases are caused by endogenous microorganisms, which express their pathogenicity when they find conditions that favor their proliferation. In order to determinate the profile of oral health and the presence of Candida in the oral cavity, clinical examination, anamnese and the samples of saliva were performed in 233 patients that asked for dental care in the clinics of UEPG. From 233 patients, 149 were female and 84 males, with mean age of $33.21 \pm 12.30$ years old. The main complaints were "treatment" and pain. The clinical examination reveled that $80 \%$ of these patients had the oral hygiene considered regular or deficient by the examiners. Candida was recovered from the oral cavity of $51.50 \%$ of the patients and in higher number in those wearing dental prosthesis. The results showed that the level of oral hygiene is low in the population studied; they only ask for dental care when infections as caries and periodontal diseases are already installed. The study also showed that the risk of development of opportunistic infections, as candidosis, is high. It's necessary the
\end{abstract}


adoption of rules to give light and stimulate the population in the maintenance of oral health.

Key words: Candida; Candidosis; oral health

Endereço para contato: e-mail: betebrasil@uol.com.br

\section{REFERÊNCIAS}

1 ARENDORF, T.M., WALKER, D.M. The prevalence and intra-oral distribution of Candida albicans in man. Arch. Oral Biol., v.5, p.1-10, 1980.

2 ARENDORF, T.M.; WALKER, D.M. Tabaco smoking and denture wering as local aethiological factors in median rhomboid glossitis. Int. J. Oral Surg., v.13, n.5, p.411-415, 1984.

3 BASTIAN, R.J.; READE, P.C. The prevalence of Candida albicans in the mouth of tabaco smokers with and without mucous membrane keratosis. Oral Surg.Oral Med. Oral Pathol., v.53, n.2, p.148-151, 1982.

4 BERDICEVSKY, H.; BEN-ARYEH, H.; SZARGEL, R. Oral Candida in asymptomatic denture wearers. Int. J. Oral Surg., v.9, n.2, p.113-115, 1980.

5 BILLINGS, R.J.; PROSKIN, H.M.; MOSS, M.E. Xerostomia and associated factors in a community-dwelling population. Communit Dent. Oral Epidemiol., v.24, n.5, p. 312-316, 1996.

6 BLAIR, Y., BAGG, J., MacFARLANE, T. W. \& CHESTNUTT, I. Microbiological assessment of denture hygiene among patients in longstay and daycare community places. Community Dent. and Oral Epidemiol., v.23, p.100-103, 1995.

7 BUDTZ-JÖRGENSEN, E. Ethiology, pathogenesis, therapy and prophylaxis of oral yeasts infections. Acta Odontol. Scand., v.48, p.61-9, 1990.

8 BUDTZ-JÖRGENSEN, E., STENDERUP, A., GRAABOWSKI, M. An epidemiologic study of yeasts in elderly denture weares. Community Dent. Oral Epidemiol., v.3, p.1159, 1975.

9 BUDTZ-JORGENSEN, E.; LOE, H. Chlorexidine as a denture disinfectant in the treatment of denture stomatitis. Scand. J. Dent. Res., v.80, n.6, p.457-464, 1972. 
10 BURFORD-MASON, A.P.; WEBER, J.C.P.; WILLOUGHBY, J.M.T. Oral carriage of Candida albicans, ABO blood group and secretor status in health patients. J. Med. Vet. Mycol., v.26, n.1, p. 46-56, 1988.

11 CROCKETT, D. N., O'GRADY, J. F. \& READE, P. C. Candida species and Candida albicans morphotypes in erythematous candidiasis. Oral Surg. Oral Med. Oral Pathol., v.73, p.559-563, 1992.

12 FOTOS, P.G., HELLSTEIN, J.W., VINCENT, S.D. Oral candidosis revisited. Gen. Dent., v.39, p.422-30, 1991.

13 GERGELY, L; URI, J. Oral micotic flora in healthy mouth. Arch. Oral Biol., v.3, n.2, p.125-128, 1961.

14 HEIMDAHL, A., NORD, C.E. Oral yeasts infections in immunocompromised and seriously diseased patients. Acta Odontol. Scand., v.48: 77-84, 1990.

15 HOLMSTRUP, P. Tabaco and oral candidosis. Abstracts of the EU- Working Group on Tabacco and Oral Health Consensus meeting. Oral Dis., v.4, n., p.53-54, 1998.

16 HOLMSTRUP, P., AXÉLL, T. Classification and clinical manifestations of oral yeast infections. Acta Odontol. Scand., v.48, p.57-9, 1990.

17 HOYEN-CHUNG, D.J. Oral hygiene training programmes in long stay hospitals. Br. Dent. J., v.167, n.5, p.178-179, 1989.

18 IACOPINO, A.M., WATHEN, W.F. Oral candidal infection and denture stomatitis: a comprehensive review. J. Am. Dent. Assoc., v.123, p.46-51, 1992.

19 KINDELAN, S.A.; YEOMAN, C.M.; DOUGLAS, C.W.I.; et. al. A comparison of intraoral carriage of Candida in Sjogren's syndrome patients with healthy xerostomic controls. Oral Surg. Oral Med. Oral Pathol., v.85,n.2, p.162167,feb.1998.

20 KULAK, Y., ARIKAN, A. \& KAZAZOGLU, E. Existence of Candida albicans and microorganisms in denture stomatitis patients. Journal of Oral Rehabilitation, v.24,p. 788-790, 1997.

21 MARSH, P.D. PERCIVAL, R.S., CHALLACOMBE, S.J. The influence of denture wearing and age on the oral microflora. J. Dent. Res., v.71, p.1374-81, 1992.

22 MILLER, C.S.; KAPLAN, A.L.; GUEST, G.F. Documenting medication use in adult dental patients. J. Am. Dent. Assoc., v.123, n.11, p.40-48, 1992.

23 NANETTI, A.; STANCARI, F.; FERRI, M. Relationship between Candida albicans and denture stomatitis: a clinical and microbiological study. New Microbiol., v.16, n.3, p.287-291, 1993. 
24 NARHI, T.O.; AINAMO, A.; MEURMAN, J.H. Salivary yeasts, saliva and oral mucosa in the elderly. J. Dent. Res., v.72, n.6, p.1009-1014, jun.1993.

25 NARHI, T.O.; MEURMAN, J.H.; AINAMO, A. Association between salivary flow rate and the use of systemic medication among 76-81 and 86-year-old inhabitants in Helsinki, Finland. J. Dent. Res., v.71, n.12, p. 1875-1880, 1992.

26 NARHI, T.O.; TENOVUO, J.; AIMANO, A. Antimicrobial factors, sialic acid, and protein concentration in whole saliva of the elderly. Scand. J. Dent. Res., v.102, n.2, p.120-125, 1994.

27 NAVAZESH, M.; CHRISTENSEN,C.; BRIGHTMAN, V. Clinical criteria for diagnosis of salivary gland hypofunction. J. Dent. Res., v.71, n.7, p. 1363-1369, 1992.

28 NEDERFORS, T.; ISAKSSON, R.; MORNSTAD, H. Prevalence of perceived symptoms of dry mouth in adult Swedish population. - relation to age, sex and pharmacotherapy. Community Dent. Oral Epidemiol., v.25, n.3, p.332-337, 1997.

29 OHMAN, S.C.; OSTERBERG, T.; DAHLEN, G. the prevalence Staphylococcus aureus, Enterobacteriaceae species and Candida species and their relationship to oral mucosal lesions in a group of 79-year-old in Goteborg. Acta Odontol. Scand., v.53, n.1, p.49-54, 1995.

30 OLIVER, D.E., SHILLITOE, E.J. Effects of smoking on the prevalence and intraoral distribution of Candida albicans. J. Oral Pathol., v.13, p.265-70, 1984.

31 PARVINEN, T.; LARMAS, M. Age dependency of stimulated salivary flow rate, pH and Lactobacillus and yeasts concentrations. J. Dent. Res., v.61, n.9, p.10521055, 1982.

32 PIETROKOVSKI, J.; AZUELOS, J.; TAU, S. Oral findings in elderly nurseing home residents in selected coutries: oral higiene conditions and plaque acumulation on denture surfaces. J. Prosthet. Dent., v.73, n.2, p.136-141, 1995.

33 RAMIREZ-AMADOR, V.; SILVERMAN, S. Jr.; MAYER, P.; MAYER, P.; TYLER, M.; QUIVEY, J. Candidal colonization and oral candidiasis in patients undergoing oral and pharyngeal radiation therapy. Oral Surg. Oral Med. Oral Pathol. Oral Radiol Endol., v.84, p.149-53, 1997.

34 RINDUM, J.L.; STENDERUP, A.; HOLMSTRUP, P. Identification of Candida albicans types related to health and pathologycal oral mucosa. J. Oral Pathol. Med., v.23, n.9, p.406-412, 1994.

35 SAKKI, T.K.; KNUUTTILA, M.L.E.; LAARA, E. The association of yeasts and denture stomatitis with behavioral and biological factors. Oral Surg. Oral Med. Oral Pathol., v.84, n.6, p.624-629, 1997. 
36 SANDVÉN, P. Laboratory identification and sensitivity testing of yeast isolates. Acta Odontol. Scand. V.48, p.27-36, 1990.

37 STENDERUP, A. Oral mycology. Acta Odontol Scand., v.48, p.3-10, 1990.

38 VISSINK, A.; SPIJKERVET, F.K.L.; AMERONGEN, A.V. Aging and saliva: a review of literature. Spec. Care Dent. V.16, n.3, p.95-103, 1996.

39 WEBB, B. C., THOMAS, C. J., WILLCOX, M. D. P., HARTY, D. W. \& KNOX, K. W. Candida-associated denture stomatitis. Aetiology and management: a review. Part 1. Factors influencing distribution of Candida species in the oral cavity. Australian Dental Journal, v. 43, p.45-50, 1998.

40 WILLIANSON, J.J. Diurnal variation of Candida albicans counts in saliva. Aust. Dent. J., v.17, n.1, p.54-60, 1972. 